\title{
Image-guided high-dose-rate interstitial brachytherapy technique for locally recurrent rectal cancer in perineum
}

\author{
Yuting Gao, BS*, Ning Wu, MD*, Zhipeng Zhao, MS, Mingyuan He, MD, Jie Han, MM, Guanghui Cheng, MD, PhD \\ Department of Radiation Oncology. China-Japan Union Hospital of Jilin University, Changchun, China \\ *These two authors contributed equally to this work
}

\begin{abstract}
Purpose: The aim of the report was the evaluation of application with image-guided high-dose-rate (HDR) interstitial brachytherapy in patients undergoing conventional chemo-radiotherapy for perineal locally recurrent rectal cancer (LRRC).

Material and methods: 75-year-old female patient presented with LRRC three years after total mesorectal excision (TME) surgery for rectal cancer with tubular adenocarcinoma (stage IIIB). Despite conventional chemotherapy and external beam radiotherapy, the re-recurrent lesion expanded as $4.5 \times 5.5 \mathrm{~cm}^{2}$ located in perineum with skin invasion. The loco-regional recurrent tumor was treated with HDR interstitial brachytherapy under ultrasound guidance and magnetic resonance imaging-based treatment planning. The brachytherapy dose was $50 \mathrm{~Gy}$ in 10 fractions of $5 \mathrm{~Gy}$ each for 5 weeks.

Results: Removal of the perineal LRRC was securely achieved by image-guided HDR interstitial brachytherapy technique. The refractory tumor healed uneventfully after interstitial brachytherapy without recurrence during 26 months of follow-up. The patient had good quality of life without serious complications of treatment.

Conclusions: Image-guided HDR interstitial brachytherapy in selected patient with LRRC in perineum is a proven, effective, and safe treatment method with relatively long-term outcome.

Key words: high-dose-rate interstitial brachytherapy, perineal recurrence, rectal cancer.

\section{Purpose}

Rectal cancer is an important cause of cancer-related deaths worldwide $[1,2,3]$. The goal in rectal cancer treatment is to optimize disease-free and overall survival, while minimizing the risk of locally recurrent rectal cancer (LRRC) $[4,5,6,7]$. LRRC is defined as recurrence of rectal cancer within the pelvis after previous standard treatment [8]. A wide range of LRRC rates after operation for rectal cancer are reported [9]. The main determinants of LRRC are related to the tumor and to the treatment [10]. Despite significant progress in treatment of rectal cancer, LRRC remains another important cause of failure of initial treatment in addition to distant metastases [11].

Management of LRRC is still unsatisfactory and re-resection is feasible only in less than $10 \%$ of cases [10]. Literature on the management of LRRC for the perineal region after chemo-radiotherapy is scarce. Brachytherapy with high dosage to target volume and restriction of normal tissue exposure may play a meaningful role in the treatment of LRRC. Although patients with aggressive lateral wall invasion or sacral invasion are contraindicated for this treatment, the LRRC might be indicated for salvage high-doserate (HDR) interstitial brachytherapy, when the patients desire to receive a treatment without re-resection [12]. Therefore, HDR interstitial brachytherapy is a promising treatment because the concentrated dose delivered to the tumor is extremely high in comparison with that delivered by external beam radiotherapy. To our knowledge, there have been few reports on the use of image-guided HDR interstitial brachytherapy for perineal LRRC, and none of those studies have involved long-term follow-up. In this context, we propose a technique using image-guided HDR interstitial brachytherapy to treat refractory perineal LRRC after conventional chemo-radiotherapy.

\section{Case description}

We encountered a 75-year-old female patient with perineal LRRC complaining of pain in the lower abdomen 
after radical surgery and chemo-radiotherapy. Eight years before being admitted to our department, she complained of difficulty with defecation and constipation and visited a vicinity clinic. On digital rectal examination and colonoscopy, hard induration and stenosis of the rectum, with an extraluminal mass $8 \mathrm{~cm}$ proximal to the anal verge was detected. Colonoscopy-guided biopsy demonstrated adenocarcinoma in June 2006. She was diagnosed with rectal cancer and received TME surgery (low anterior resection - LAR). The histopathological result after surgery showed that tubular adenocarcinoma $\left(5.0 \times 4.0 \times 1.0 \mathrm{~cm}^{3}\right)$ invaded through the muscularis propria of rectum with regional lymph nodes (2/15) metastasis (pT3N1M0; IIIB stage according to the $6^{\text {th }}$ edition of the American Joint Committee on Cancer/Union for International Cancer Control - AJCC/UICC [13]). Then the patient was treated with adjuvant chemotherapy with 5-fluorouracil (5-FU) immediately for 2 cycles. Three years after the initial TME, the patient noticed a dull pain in the perineum. In July 2009, abdominal ultrasonography showed an irregular low signal intensity mass $\left(5.3 \times 4.2 \mathrm{~cm}^{2}\right)$ located between the rectum and posterior vaginal wall. A diagnostic biopsy revealed a locoregional recurrent malignant tumor (originating from the rectum). The patient refused surgery treatment. Chemotherapy treatment using XELOX regimen (capecitabine plus oxaliplatin) was carried out every 3 weeks with a total of 5 cycles. The patient therefore underwent initial external beam radiotherapy with intensity modulated radiation therapy (IMRT) technique at the local hospital in January 2010. The clinical target volume (CTV)- ${ }_{\text {IMRT }}$ included the entire mesorectum, presacral and internal iliac nodal regions. The planning target volume (PTV) ${ }_{\text {IMRT }}$ was defined adding three-dimensional $7 \mathrm{~mm}$ margins to the CTV- ${ }_{\text {IMRT }}$. Prescription doses were $50 \mathrm{~Gy}$ to CTV-IMRT and $60 \mathrm{~Gy}$ to the gross target volume (GTV)- ${ }_{\text {IMRT }}$ at 2 Gy/fractions, all given in 6 weeks. The following values were accepted: the volume ratios of bladder, sacrum, and femoral head receiving more than 50 Gy were $22.02 \%, 23.94 \%$, and $0.51 \%$, respectively; the volume of bowel receiving more than 45 Gy was $14.41 \mathrm{~cm}^{3}$. During the external radiotherapy, the concurrent chemotherapy (capecitabine) was performed and

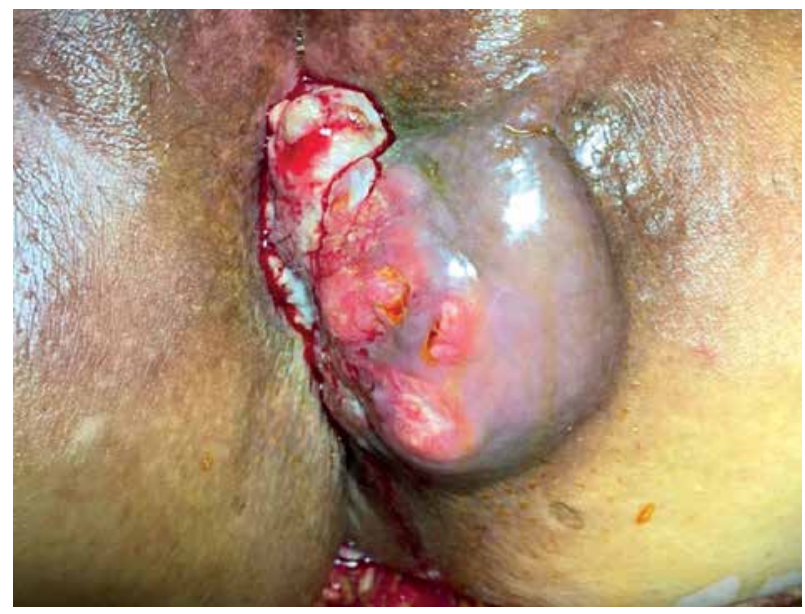

Fig. 1. Recurrent tumor in the perineum the tumor shrank gradually. The patient's condition remained stable for five years until a complaint of oppressive pain with a hard mass in the perineum in March 2015. She had an abnormal magnetic resonance imaging (MRI) scan revealing an area of distortion of approximately $4.5 \times$ $5.5 \mathrm{~cm}^{2}$ in size $5 \mathrm{~cm}$ proximal to the anal verge. The tumor invaded the perineal skin surface with local ulceration (Figure 1). The blood biomarker tests confirmed obvious elevation of carcinoembryonic antigen (CEA), carbohydrate antigen 199 and 242 (CA 199 and CA 242). Furthermore, the distant metastatic lesions could not be identified according to conventional examination. Despite recommendation for subsequent palliative resection, the patient refused surgery. She was referred to our clinic for treatment and accepted the suggestion of brachytherapy.

\section{Treatment modalities}

Before processing the treatment, an informed consent was obtained from the patient. Treatment were performed with standard institutional approval. HDR interstitial brachytherapy of 50 Gy in 10 fractions for 5 weeks (5 Gy each fraction, twice a day with 6 hours interval, 2 fractions per week, 5 weeks) was applied. The treatment was accomplished with titanium interstitial needles (Nucletron, an Elekta company, Elekta AB, Stockholm, Sweden) and performed under anesthesia. Electrocardiogram, arterial oxygen pressure, respiration, and blood pressure monitoring were performed during the procedure. Fifteen needle applicators $(1.9 \mathrm{~mm}$ in external diameter and $20 \mathrm{~cm}$ in length) were transdermally inserted to the target at the different depth under transvaginal ultrasound guidance, and simultaneously controlling the position of the needle against the adjacent structures. The applicators were inserted in such a way that their layout in the lesion was possibly most parallel, and their distance from one another was nearly $1 \mathrm{~cm}$ to ensure adequate dose distribution and target volume coverage (Figure 2).

After implanting the needle applicators, they remained in the lesion throughout the treatment process, and the 3.0T MRI were then acquired and transferred to the treatment planning computer. The MRI data of interstitial bra-

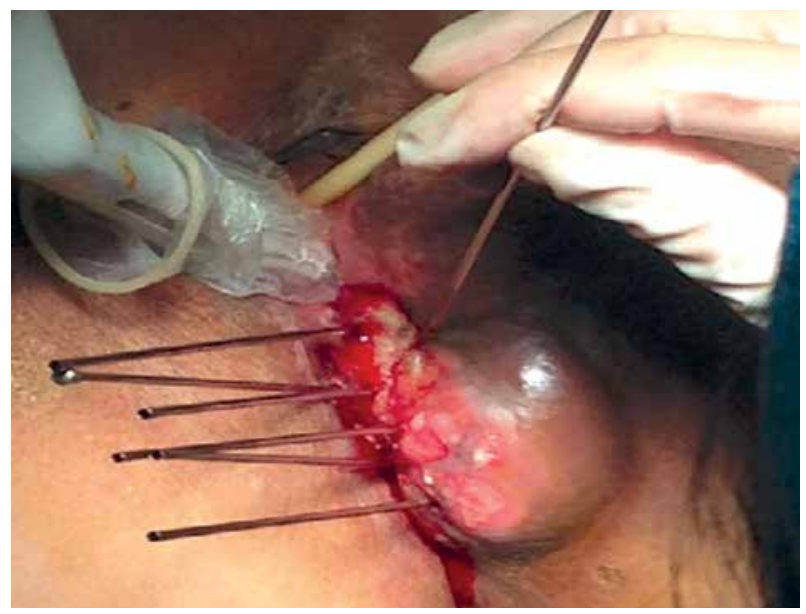

Fig. 2. Needle applicators inserted to the target under ultrasound guidance 

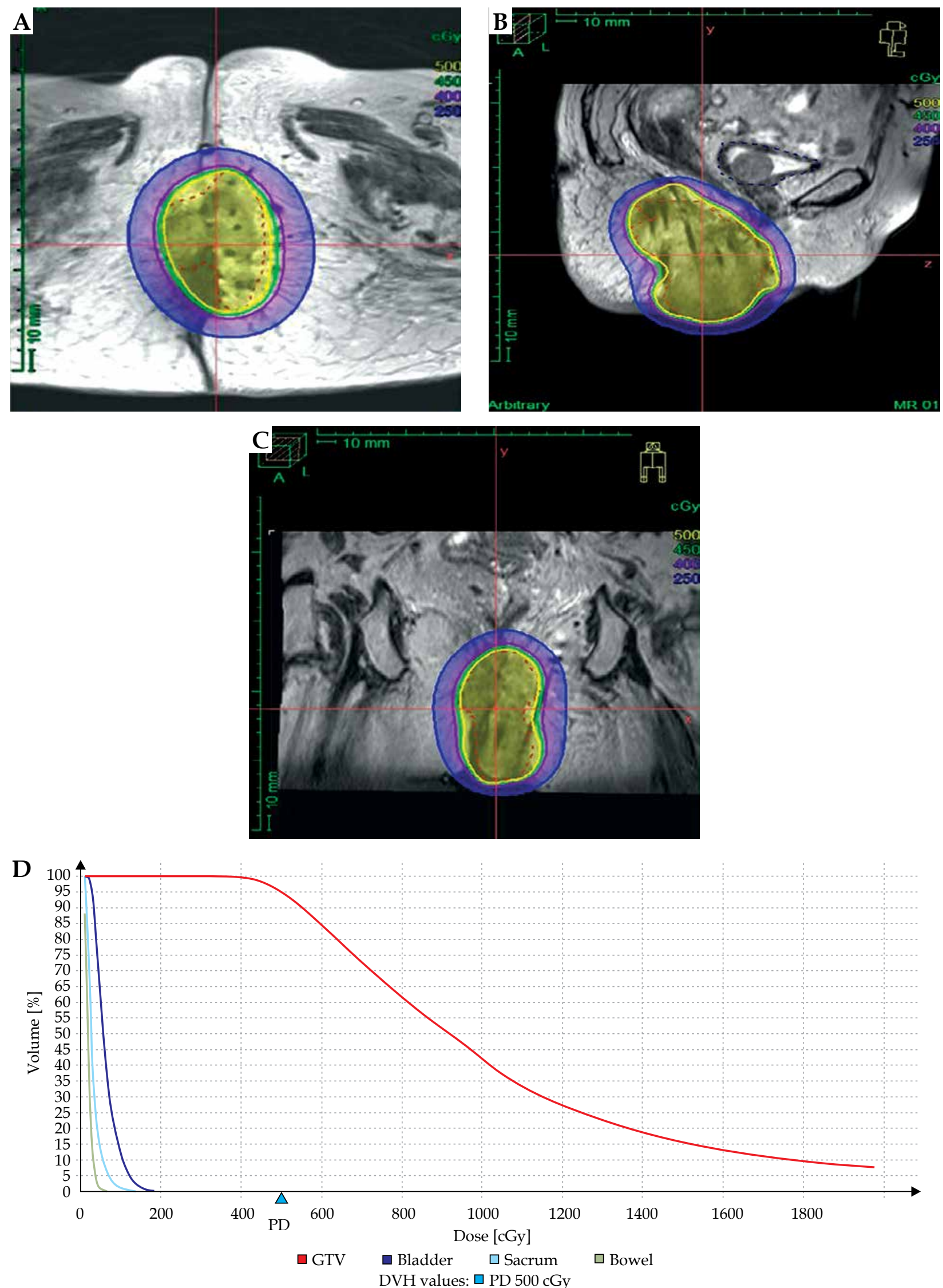

Fig. 3. The dose distribution of A) horizontal, B) sagittal, and C) coronal positions. The dose volume histogram parameters in a treatment session (D) 
chytherapy was used to contour the GTV- ${ }_{-\mathrm{BT}}$ and CTV- ${ }_{\mathrm{BT}}$. The CTV- ${ }_{\text {BT }}$ was expanded from GTV- ${ }_{-\mathrm{BT}}$ by $10 \mathrm{~mm}$ and restricted by the volume of organs at risk (OAR), which included the bladder, sacrum, and bowel. MRI-based treatment plan was created using a graphic optimization tool (treatment planning system Oncentra version 4.3; Nucletron, an Elekta company, Elekta AB, Stockholm, Sweden). The normalization and optimization to the target volume was performed (Figure 3 A-C).

The dose volume histogram (DVH) parameters are recommended for the evaluation of target volume and OAR. The dose distribution to target volume and OAR are presented in Figure 3D. In the brachytherapy plan, 50 Gy was prescribed to $100 \%$ of the target volume. $\mathrm{D}_{2 \mathrm{cc} \mathrm{c}^{\prime}} \mathrm{D}_{1 \mathrm{cc} \mathrm{c}^{\prime}}$ and $\mathrm{D}_{0.1 \mathrm{cc}}$ (minimum dose to the most irradiated volume of 2,1 , and $0.1 \mathrm{ml}$, respectively) of the bladder, sacrum, and bowel were mainly considered. The equivalent dose for 2 Gy fraction schedule was calculated using $\mathrm{EQD}_{2}$ model; at $\alpha / \beta=3\left(\mathrm{~Gy}_{\mathrm{EQD} 2, \alpha / \beta=3}\right)$ for the OAR, and $\alpha / \beta=10$ $\left(\mathrm{Gy}_{\mathrm{EQD} 2, \alpha / \beta=10}\right)$ for the target. DVH parameters were analyzed taking into account the volume ratio of the target receiving $90 \%, 100 \%$, or $150 \%$ of the therapeutic dose $\left(\mathrm{V}_{90 \%}, \mathrm{~V}_{100 \%}\right.$, and $\mathrm{V}_{150 \%}$, respectively); the equivalent dose delivered to $98 \%, 90 \%$, and $50 \%$ of the target volume $\left(\mathrm{D}_{98 \%}\right.$, $\mathrm{D}_{90 \%}$, and $\mathrm{D}_{50 \%}$, respectively). These doses were converted according to linear quadratic model of biologic effective dose (BED). Dose homogeneity index (DHI), which was used to evaluate the dose uniformity of the target (DHI $=1-V_{150 \%} / V_{100 \%}$ ) was calculated for single fraction.

After transporting planning data to an iridium-192 ( ${ }^{192} \mathrm{Ir}$ ) remote afterloader system (Microselectron HDR ${ }^{192}$ Ir; Nucletron, an Elekta company, Elekta AB, Stockholm, Sweden), the irradiation started and took approximately $5 \mathrm{~min}$. Needle applicators were located in the lesion only during each application (included 2 fractions in a day, with 6 hours interval, 5 treatment applications in 5 weeks) of treatment and removed after irradiation was completed. Due to abundant vascularity of perineum, there was a small degree of bleeding at the time of needle applicators removal, which was resolved spontaneously. No hemorrhagic foci were found on MRI scans taken after the insertion and removal of applicators. No clinical features of internal hemorrhage were observed. The patient was discharged after 2 hours of observation.
No complications were reported during the treatment, and brachytherapy was well tolerated by the patient. The patient is regularly followed up at our affiliated clinics.

\section{Results}

In this patient, needle applicators were used, and the insertion and MRI-based treatment plan were performed before each treatment application. Due to this fact, fractional doses were higher (5 Gy each fraction) and the interval between sessions was longer ( 7 days). $\mathrm{D}_{98 \%}$ median of a single fraction was $4.43 \mathrm{~Gy}$ (range, 4.03-4.73 Gy), $\mathrm{D}_{90 \%}$ median of a single fraction was 5.49 Gy (range, 5.06-5.67 Gy), and $\mathrm{D}_{50 \%}$ median of a single fraction was 8.39 Gy (range, 7.53-9.20 Gy). CTV-вт received $90 \%$ dose as well as $100 \%$ and $150 \%$ dose (Table 1). DHI was calculated for each application and it median was 0.36 (range, $0.29-0.44$ ). Depending on the location of irradiated area, doses received by OAR were reported (Table 2).

During brachytherapy, it could be observed that the recurrent tumor in perineum shrank gradually between subsequent treatment applications with slight radiation-induced skin reaction (RTOG scale grade 1), which was characterized by redness and pigmentation. The target volume at the time of the last treatment application was reduced from $159.63 \mathrm{~cm}^{3}$ (before brachytherapy) to $108.98 \mathrm{~cm}^{3}$ (Figure 4).

During follow-up examination in the $3^{\text {rd }}$ month after brachytherapy, it was showed that the perineal mass disappeared with normal CEA, CA 199, and CA 242 (Figure $5 \mathrm{~A}$ ). The related region of skin surface was basically recovered, and the pain symptoms decreased. At the present 26 months after brachytherapy, treatment-related skin side effect has completely healed (Figure 5B). In addition, there are no signs or symptoms of complications and no evidence of recurrence at the site of HDR interstitial brachytherapy.

\section{Discussion}

Advances in the management of rectal cancer with therapeutic modalities have improved prognosis and survival of this type of cancer [14]. Despite improvements in both the neoadjuvant and surgical management of rectal

Table 1. Doses of $D_{98 \%}, D_{90 \%}$, and $D_{50 \%}$, value of $V_{90 \%}, V_{100 \%}, V_{150 \%}$, and dose homogeneity index (DHI) for target volume

\begin{tabular}{lcccccccc} 
& $\mathrm{GTV}$ volume $\left(\mathrm{cm}^{3}\right)$ & $\mathrm{D}_{98 \%}(\mathrm{~Gy})$ & $\mathrm{D}_{90 \%}(\mathrm{~Gy})$ & $\mathrm{D}_{50 \%}(\mathrm{~Gy})$ & $\mathrm{V}_{90 \%}(\%)$ & $\mathrm{V}_{100 \%}(\%)$ & $\mathrm{V}_{150 \%}(\%)$ & $\mathrm{DHI}$ \\
\hline $\mathrm{BT}_{1,2}$ & 159.63 & 4.54 & 5.52 & 9.20 & 98.21 & 94.96 & 67.12 & 0.29 \\
\hline $\mathrm{BT}_{3,4}$ & 133.20 & 4.73 & 5.56 & 8.57 & 99.06 & 96.14 & 62.74 & 0.35 \\
\hline $\mathrm{BT}_{5,6}$ & 132.66 & 4.36 & 5.67 & 8.18 & 97.57 & 95.44 & 59.43 & 0.38 \\
\hline $\mathrm{BT}_{7,8}$ & 112.04 & 4.03 & 5.06 & 7.53 & 95.27 & 90.64 & 50.37 & 0.44 \\
\hline $\mathrm{BT}_{9,10}$ & 108.98 & 4.48 & 5.66 & 8.47 & 97.96 & 96.32 & 61.97 & 0.36 \\
\hline $\mathrm{EQD}_{2-\text { amount }}$ & - & 53.27 & 70.98 & 128.82 & - & - & - & - \\
\hline BED-amount & - & 63.93 & 85.17 & 154.59 & - & - & - & -
\end{tabular}

$D_{98 \%}, D_{90 \%}, D_{50 \%}$ - dose covering $98 \%, 90 \%$, and $50 \%$ of target volume; $V_{90 \%}, V_{100 \%}, V_{150 \%}$ - percentage of target volume receiving $90 \%, 100 \%$, and $150 \%$ of prescription dose; $D H I$ - dose homogeneity index; BT - fractions of brachytherapy; $E Q D_{2^{-}}$amount - dose equivalent 2 Gy for total fractions; $B E D$-amount - biologic effective dose for total fractions 
cancer [15], LRRC, which occurs in 3\% to over $30 \%$ according to this publication [10], is still the most important factor determining prognosis and survival apart from distant metastases. For rectal cancer, the most common recurrent location is at or around the anastomosis and presacral region, including pelvic or perineal lesions [16]. Different treatments such as surgery, radiation, chemotherapy, hyperthermia, or combinations have been used

Table 2. Doses (Gy) in organs at risk (OAR): bladder, sacrum, and bowel

\begin{tabular}{lccccccccc} 
& $\begin{array}{c}\text { Bladder- } \\
\mathrm{D}_{2 c c}\end{array}$ & $\begin{array}{c}\text { Bladder- } \\
\mathrm{D}_{1 c c}\end{array}$ & $\begin{array}{c}\text { Bladder- } \\
\mathrm{D}_{0.1 c c}\end{array}$ & $\begin{array}{c}\text { Sacrum- } \\
\mathrm{D}_{2 c c}\end{array}$ & $\begin{array}{c}\text { Sacrum- } \\
\mathrm{D}_{1 c c}\end{array}$ & $\begin{array}{c}\text { Sacrum- } \\
\mathrm{D}_{0.1 c c}\end{array}$ & $\begin{array}{c}\text { Bowel- } \\
\mathrm{D}_{2 c c}\end{array}$ & $\begin{array}{c}\text { Bowel- } \\
\mathrm{D}_{1 c c}\end{array}$ & $\begin{array}{c}\text { Bowel- } \\
\mathrm{D}_{0.1 c c}\end{array}$ \\
\hline $\mathrm{BT}_{1,2}$ & 1.60 & 1.70 & 1.93 & 0.77 & 0.89 & 1.29 & 0.47 & 0.51 & 0.68 \\
\hline $\mathrm{BT}_{3,4}$ & 1.34 & 1.44 & 1.67 & 0.65 & 0.79 & 1.37 & 0.57 & 0.62 & 0.78 \\
\hline $\mathrm{BT}_{5,6}$ & 1.53 & 1.65 & 1.89 & 0.98 & 1.15 & 1.77 & 0.60 & 0.66 & 0.83 \\
\hline $\mathrm{BT}_{7,8}$ & 1.43 & 1.55 & 1.81 & 0.77 & 0.89 & 1.38 & 0.61 & 0.67 & 0.84 \\
\hline $\mathrm{BT}_{9,10}$ & 1.60 & 1.73 & 1.98 & 1.21 & 1.47 & 2.17 & 0.65 & 0.69 & 0.87 \\
\hline $\mathrm{EQD}_{2-\text { amount }}$ & 13.51 & 14.92 & 18.05 & 6.87 & 8.50 & 14.89 & 4.15 & 4.50 & 6.10 \\
\hline $\mathrm{BED}_{\text {-amount }}$ & 22.54 & 24.86 & 30.07 & 11.46 & 14.18 & 24.82 & 6.94 & 7.64 & 9.89
\end{tabular}

Bladder/Sacrum/Bowel- $D_{2 c c}, D_{1 c c}$ and $D_{0.1 c c}$ - minimum dose to the most irradiated volume of $2 \mathrm{ml}, 1 \mathrm{ml}$, and $0.1 \mathrm{ml}$ of the bladder, sacrum, and bowel; $B T$ - fractions of brachytherapy; $E Q D_{2-}$-amount - dose equivalent $2 \mathrm{~Gy}$ for total fractions; $B E D$-amount - biologic effective dose for total fractions
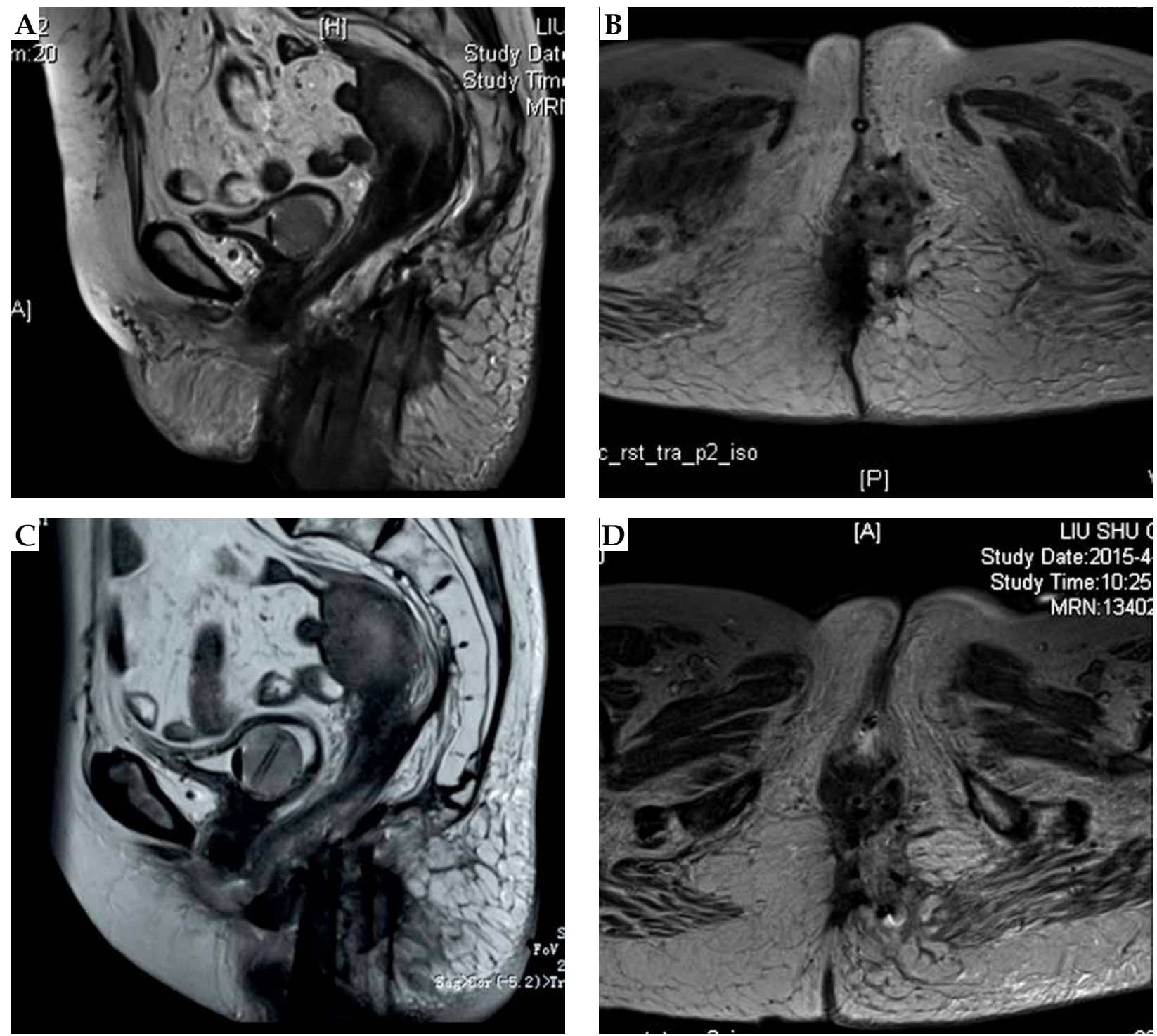

Fig. 4. The target volume in (A, B) first and (C, D) last treatment applications of image-guided high-dose-rate interstitial brachytherapy 

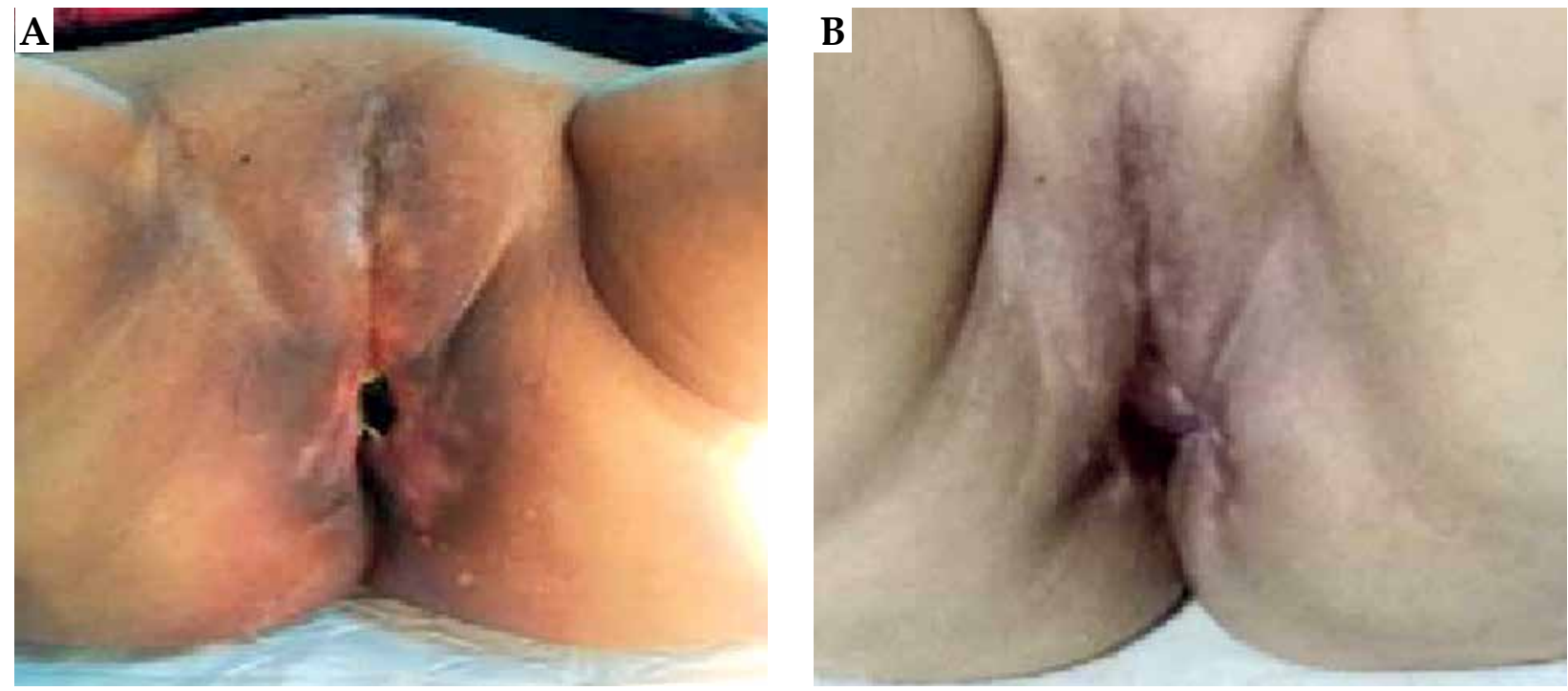

Fig. 5. The skin surface of tumor related region A) 3 months, and B) 26 months after image-guided high-dose-rate interstitial brachytherapy

for LRRC, but an optimal treatment schedule remains unproven $[17,18]$. Some studies reported high re-recurrence rate of patients with LRRC who underwent surgical resection, and the surgical outcomes of patients who underwent surgery for re-recurrent rectal cancer are still unsatisfactory $[19,20,21]$. Although chemo-radiotherapy could be a potential option for LRRC cure [22], Sun et al. [23] investigated that the complete response rate was only $8.3 \%$ and symptomatic responses proved to be obvious. Local re-recurrences impose a major problem in patients with LRRC who received intraoperative radiotherapy (47\%, 5-year rate), as also described by Kusters et al. [24]. Milani et al. [25] published results of phase II studies about a multimodal salvage therapy including radiochemotherapy and regional hyperthermia in pre-irradiated patients with LRRC. The median local progression-free survival was 15 months, and grade 3 gastrointestinal acute toxicity were observed in $12.5 \%$ of the patients. Brachytherapy was not established as a standard therapy for LRRC but some explorative researches have focused on its clinical application [12].

Because of the particularity of the anatomic site, there are few reports on the therapeutic experience of perineal LRRC. Zhao et al. [26] investigated the feasibility of re-resection in a patient with perineal LRRC that recurrent many times and received 12 surgeries during 3 years. Although adjuvant radiation therapy and chemotherapy were also carried out, the quality of life after treatment for patient with perineal LRRC was far from satisfactory. In the present case, the image-guided HDR interstitial brachytherapy was used with good adherence in order to control the perineal recurrent tumor. The DVH showed that target volume was covered with high-dose irradiation, but the dosage of OAR was relatively low. The patient with refractory recurrence obtained good loco-regional control by the HDR interstitial radiotherapy with ultrasound guidance and MRI-based treatment planning. The tumor shrank visibly 3 months later, and no serious complications were found in follow-up over 2 years.

\section{Conclusions}

The main benefit of HDR interstitial brachytherapy is that a high-dose of radiation can be precisely applied to the tumor, while sparing radiation to healthy tissues simultaneously. Although there is ongoing discussion regarding the best management and/or optimal treatment procedure for refractory perineal LRRC, the image-guided HDR interstitial brachytherapy may be a promising salvage treatment approach with tolerable toxicity in selected patients.

\section{Acknowledgements}

This study was partially supported by grants from the National Natural Science Foundation of China (81201737, 31600679), Project of Science and Technology Department of Jilin Province (20090458), Project of Health and Family Planning Commission of Jilin Province (2014ZC054), Bethune Special Research of Science and Technology Department of Jilin Province (20160101079JC), Horizontal Project of Jilin University (2015373), Health and Family Planning Commission Foundation of Jilin Province (2014ZC054), Foundation for Natural Science (Bethune Special Project) of Jilin Provincial Science and Technology Department (20160101079JC), Jilin University Enterprise Sponsored Research Foundation (2015YX154), Jilin University Network Experiment Project (VE2015081), Jilin University Undergraduate Education Reform Research Project $(2015,2017$ XYB080) and Jilin University Norman Bethune Medical Department Teaching Reform Research Project (B2014B137).

\section{Disclosure}

The authors report no conflict of interests.

\section{References}

1. Siegel RL, Miller KD, Jemal A. Cancer statistics, 2015. CA Cancer J Clin 2015; 65: 5-29. 
2. Gaertner WB, Kwaan MR, Madoff RD et al. Rectal cancer: an evidence-based update for primary care providers. World I Gastroenterol 2015; 21: 7659-7671.

3. Global Burden of Disease Cancer Collaboration, Fitzmaurice C, Dicker D, Pain A et al. The global burden of cancer 2013. JAMA Oncol 2015; 1: 505-527.

4. De Cauwé L, Van Nieuwenhove Y, Ceelen WP. Preoperative chemoradiation versus radiation alone for stage II and III resectable rectal cancer. Cochrane Database Syst Rev 2013; 2: CD006041.

5. Martin ST, Heneghan HM, Winter DC. Systematic review and meta-analysis of outcomes following pathological complete response to neoadjuvant chemoradiotherapy for rectal cancer. Br J Surg 2012; 99: 918-928.

6. Maas M, Nelemans PJ, Valentini V et al. Long-term outcome in patients with a pathological complete response after chemoradiation for rectal cancer: a pooled analysis of individual patient data. Lancet Oncol 2010; 11: 835-844.

7. Kosinski L, Habr-Gama A, Ludwig K et al. Shifting concepts in rectal cancer management: a review of contemporary primary rectal cancer treatment strategies. CA Cancer J Clin 2012; 62: 173-202.

8. PelvEx Collaborative. Factors affecting outcomes following pelvic exenteration for locally recurrent rectal cancer. Br J Surg 2018; 105: 650-657.

9. Bouchard P, Efron J. Management of recurrent rectal cancer. Ann Surg Oncol 2010; 17: 1343-1356.

10. Roncucci L. Local recurrences of rectal cancer. Minerva Chir 2005; 60: 167-178.

11. Díaz Beveridge R, Akhoundova D, Bruixola G et al. Controversies in the multimodality management of locally advanced rectal cancer. Med Oncol 2017; 34: 102.

12. Morimoto M, Isohashi F, Yoshioka Y et al. Salvage high-doserate interstitial brachytherapy for locally recurrent rectal cancer: long-term follow-up results. Int J Clin Oncol 2014; 19: 312-318.

13. Sobin L, Wittekind C. TNM classification of malignant tumors. Wiley-Liss, New York 2002.

14. Ben Abid S, Hefaiedh R, Mzoughi Z et al. What's new in rectal cancer? Tunis Med 2014; 92: 593-600 [Article in French].

15. Montrone S, Cantarella M, Coraggio G et al. Preoperative short course radiotherapy in elderly patients ( $\geq 75$ years) affected by locally advanced rectal cancer. Eur J Cancer 2015; 51: S342.

16. Di Bartolomeo N, Balestra MR, Liddo G et al. Pelvic recurrence of rectal cancer: our experience. Suppl Tumori 2005; 4: S21-23.

17. Sakurai H, Mitsuhashi N, Harashima K et al. CT-fluoroscopy guided interstitial brachytherapy with image-based treatment planning for unresectable locally recurrent rectal carcinoma. Brachytherapy 2004; 3: 222-230.

18. Harris CA, Solomon MJ, Heriot AG et al. The Outcomes and Patterns of Treatment Failure After Surgery for Locally Recurrent Rectal Cancer. Ann Surg 2016; 264: 323-329.

19. Koyama M, Murata A, Sakamoto Y et al. Surgical treatment outcomes of multimodality therapy for locally recurrent rectal cancer. Gan To Kagaku Ryoho 2014; 41: 1459-1461 [Article in Japanese].

20. Nielsen M, Rasmussen P, Pedersen B et al. Early and late outcomes of surgery for locally recurrent rectal cancer: a prospective 10-year study in the total mesorectal excision era. Ann Surg Oncol 2015; 22: 2677-2684.

21. Harji DP, Sagar PM, Boyle K et al. Outcome of surgical resection of second-time locally recurrent rectal cancer. Br J Surg 2013; 100: 403-409.

22. Lee JH, Kim DY, Kim SY et al. Clinical outcomes of chemoradiotherapy for locally recurrent rectal cancer. Radiat Oncol 2011; 6: 51 .
23. Sun DS, Zhang JD, Li L et al. Accelerated hyperfractionation field-involved re-irradiation combined with concurrent capecitabine chemotherapy for locally recurrent and irresectable rectal cancer. Br J Radiol 2012; 85: 259-264.

24. Kusters M, Dresen RC, Martijn H et al. Radicality of resection and survival after multimodality treatment is influenced by subsite of locally recurrent rectal cancer. Int J Radiat Oncol Biol Phys 2009; 75: 1444-1449.

25. Milani V, Pazos M, Issels RD et al. Radiochemotherapy in combination with regional hyperthermia in preirradiated patients with recurrent rectal cancer. Strahlenther Onkol 2008; 184: 163-168.

26. Zhao R, Zhang L. Case report: Locoregional (perineum and inguinal) recurrence after treatment of rectal cancer by low anterior resection. World J Surg Oncol 2013; 11: 186. 